\title{
Biorelevant Dissolution Media Simulating the Proximal Human Gastrointestinal Tract: An Update
}

\author{
Ekarat Jantratid and Jennifer Dressman ${ }^{1}$ \\ Institute of Pharmaceutical Technology, Johann Wolfgang Goethe University, \\ Max-von-Laue-Str. 9, 60438 Frankfurt am Main, Germany
}

\section{INTRODUCTION}

$\Lambda$ $s$ part of a drive to develop predictive in vitro models to forecast the in vivo performance of drugs and drug products, two biorelevant dissolution media simulating conditions in the proximal small intestine, Fasted-State Simulated Intestinal Fluid (FaSSIF) and Fed-State Simulated Intestinal Fluid (FeSSIF), were proposed in 1998 (1). Although these media have been proved useful in terms of in vivo predictions, there is room for improvement. One aspect that might lead to suboptimal predictions is that some parameters in FaSSIF and FeSSIF (e.g., buffer capacity) were chosen on the basis of canine, rather than human data (1). Additionally, since the first set of biorelevant media were developed, there have been several studies of the relevant parameters in humans $(2,3)$. Of particular note is that the bile salt concentrations reported in more recent studies tend to be lower than in older reports, most likely as a result of more specific analytical techniques. Moreover, the previous versions of the biorelevant media do not reflect the lipolysis products of meal digestion that are known to enhance the solubility and dissolution of poorly soluble lipophilic drugs (3).

The biorelevant dissolution media have been updated recently to bring the composition and characteristics closer to those of aspirates collected from the human gastrointestinal (Gl) tract (4). Together with the medium simulating the preprandial gastric conditions proposed by Vertzoni et al. in 2005 (5), a core group of four biorelevant media simulating both stomach and proximal small intestine of humans in the pre- and postprandial states has been established (4). These media can be used to investigate the release characteristics of drugs and drug products in the stomach and small intestine, particularly in terms of food effects. This article summarizes the media composition and their important characteristics. In addition, examples of applications of the in vivo predictiveness of these media are presented.

\section{BIORELEVANT MEDIA SIMULATING THE ENVIRONMENT IN THE STOMACH}

The stomach is the port of entry into the Gl tract for orally administered drug products. It is here that immediate-release (IR) dosage forms should disintegrate,

${ }^{1}$ Corresponding author. enabling the active pharmaceutical ingredient (API) to dissolve in a timely manner before it reaches the absorptive sites in the small intestine. Under fasting conditions, it is well known that the $\mathrm{pH}$ in a healthy human stomach is acidic, ranging between 1 and $3(2,6)$. For poorly soluble weak bases, the $\mathrm{pH}$ conditions for dissolution are the most favorable in a fasted, healthy stomach. Compared with simple aqueous buffers like Simulated Gastric Fluid without pepsin $\left(\mathrm{SGF}_{\mathrm{sp}}\right)$, gastric fluids have a low surface tension in addition to a low $\mathrm{pH}$ (7). A medium representing the fasted conditions in a human stomach, so-called Fasted-State Simulated Gastric Fluid (FaSSGF), was proposed by Vertzoni et al. in 2005 (5). The medium was designed to embrace the important aspects of human basal gastric juice plus a glass of water normally given with a dosage form. The composition and properties of FaSSGF are shown in Table 1.

With respect to the applications of FaSSGF, the medium is able to predict the solubility of poorly soluble drugs in the fasted stomach rather well $(5,8)$. Figure 1 compares the solubility of ketoconazole in human gastric aspirates with that in FaSSGF and other media. The solubility results in FaSSGF were most similar to those in the aspirates.

In the postprandial state, the stomach environment varies over the course of gastric residence of the meal (2).

Table 1. Composition of the Medium to Simulate the FastedState Stomach: Fasted-State Simulated Gastric Fluid (FaSSGF)

\begin{tabular}{lc}
\hline Composition & \\
\hline Sodium taurocholate $(\mu \mathrm{M})$ & 80 \\
\hline Lecithin $(\mu \mathrm{M})$ & 20 \\
\hline Pepsin $(\mathrm{mg} / \mathrm{mL})$ & 0.1 \\
\hline Sodium chloride $(\mathrm{mM})$ & 34.2 \\
\hline Hydrochloric acid q.s. & $\mathrm{pH} 1.6$ \\
\hline Properties & 1.6 \\
\hline pH & $120.7 \pm 2.5$ \\
\hline Osmolality $(\mathrm{mOsm} / \mathrm{kg})$ & - \\
\hline Buffer capacity $(\mathrm{mmol} / \mathrm{L} / \mathrm{pH})$ & 42.6 \\
\hline Surface tension $(\mathrm{mN} / \mathrm{m})$ &
\end{tabular}

Dissolution Technologies | AUGUST 2009 


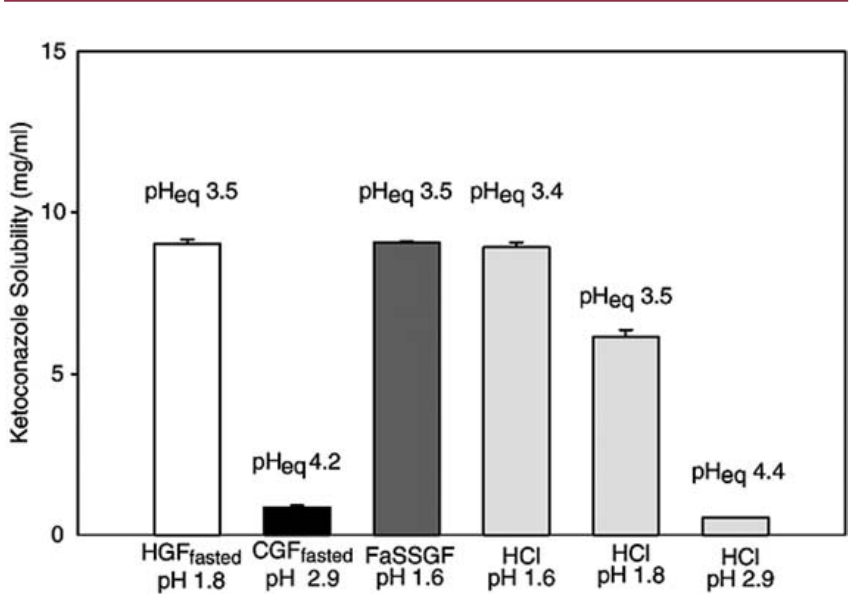

Figure 1. Mean $+S D(n=3)$ solubility data of ketoconazole in human gastric fluid (HGF), in canine gastric fluid (CGF), in Fasted-State Simulated Gastric Fluid (FaSSGF), and in $\mathrm{HCl}$ solutions with initial $\mathrm{pH}$ values 1.6, 1.8, and 2.9. $\mathrm{pH}_{\text {eq }}$ is the $\mathrm{pH}$ of the medium at equilibrium. (Reproduced with permission from ref 8. Copyright 2007 Elsevier.)

Nevertheless, it is desirable to have a global medium reflecting the changes in the stomach after meal intake to make an estimate of food effects on drug dissolution and release. Milk (full-fat [3.5\%], long-life, UHT-treated) has been considered as a good starting point for medium design because its ratio of carbohydrate/protein/fat is similar to that observed in the stomach after administration of meals (e.g., those recommended by the U.S.HHS-FDA (8) for use in bioavailability and bioequivalence studies). Three "snapshot" media representing the substantial changes in gastric conditions during the time frame of about 200 min after meal intake have been proposed (4). Among them, the middle one appears to reflect the general changes in the gastric fed state best, and it has been designated as Fed-State Simulated Gastric Fluid (FeSSGF). The compositions of three gastric snapshot media including FeSSGF are presented in Table 2. Recently, successful IVIVC has been demonstrated in a fed canine model (9) by applying FeSSGF to predict the oral absorption of an experimental Roche compound, RZ-50, a poorly soluble, weakly acidic drug formulated as a lipid-based dosage form.

\section{BIORELEVANT MEDIA SIMULATING THE ENVIRONMENT IN THE UPPER SMALL INTESTINE}

In general, the small intestine represents the region where drugs are best absorbed. Additionally, for poorly soluble, weakly acidic compounds and lipophilic compounds, dissolution is favored in this segment. The previous versions of FaSSIF and FeSSIF reflect some crucial aspects in the small intestinal milieu after meal intake, namely the bile components and $\mathrm{pH}$ (1). The media have recently been fine-tuned to bring the compositions and characteristics closer to those of the human small intestinal environment (3).
Table 2. Composition of the Media to Simulate the Fed-State Stomach, Including Fed-State Simulated Gastric Fluid (FeSSGF)

\begin{tabular}{lccc}
\hline Composition & Early & $\begin{array}{c}\text { Middle } \\
\text { (FeSSGF) }\end{array}$ & Late \\
\hline Sodium chloride (mM) & 148 & 237.02 & 122.6 \\
\hline Acetic acid (mM) & - & 17.12 & - \\
\hline Sodium acetate (mM) & - & 29.75 & - \\
\hline Ortho-phosphoric acid (mM) & - & - & 5.5 \\
\hline Sodium dihydrogen phosphate (mM) & - & - & 32 \\
\hline Milk/buffer & $1: 0$ & $1: 1$ & $1: 3$ \\
\hline Hydrochloric acid/sodium hydroxide & $\mathrm{pH} 6.4$ & $\mathrm{pH} 5$ & $\mathrm{pH} 3$ \\
q.s. & & & \\
\hline Properties & & & \\
\hline pH & 6.4 & 5 & 3 \\
\hline Osmolality (mOsm/kg) & 559 & 400 & 300 \\
\hline Buffer capacity (mmol/L/pH) & 21.33 & 25 & 25 \\
\hline Surface tension (mN/m) & $49.7 \pm 0.3$ & $52.3 \pm 0.3$ & $58.1 \pm 0.2$ \\
\hline
\end{tabular}

The composition of the updated FaSSIF, so-called FaSSIF-V2, was changed slightly to more closely reflect the in vivo data, while the buffer has been changed from phosphate to maleate for practical reasons (4).

Table 3 demonstrates the composition of FaSSIF-V2.

In the fed state, the environment in the small intestine also changes considerably after a meal compared with the fasting conditions. The same snapshot approach as used for the postprandial gastric media was also applied to the

Table 3. Composition of the Medium to Simulate the FastedState Upper Small Intestine: Fasted-State Simulated Intestinal Fluid, Updated Version (FaSSIF-V2)

\begin{tabular}{ll}
\hline Composition $(\mathrm{mM})$ & 3 \\
\hline Sodium taurocholate & 0.2 \\
\hline Lecithin & 19.12 \\
\hline Maleic acid & 34.8 \\
\hline Sodium hydroxide & 68.62 \\
\hline Sodium chloride & 6.5 \\
\hline Properties & $180 \pm 10$ \\
\hline pH & 10 \\
\hline Osmolality $(\mathrm{mOsm} / \mathrm{kg})$ & 54.3 \\
\hline Buffer capacity $(\mathrm{mmol} / \mathrm{L} / \mathrm{pH})$ & \\
\hline Surface tension $(\mathrm{mN} / \mathrm{m})$ & \\
\hline
\end{tabular}


design of postprandial small intestinal media. From the three snapshot media, a new medium representing the conditions in the proximal small intestine after meal ingestion has been designed. This updated FeSSIF medium is called FeSSIF-V2. The medium combines the postprandial changes in $\mathrm{pH}$, buffer capacity, osmolality, and bile component concentrations, while utilizing concentrations of lipolysis products that reflect aqueous phase values in the aspirates. There are lower amounts of bile components in FeSSIF-V2 than in FeSSIF, but this is compensated for by adding meal digestion products, including monoglycerides and free fatty acids, both of which can enhance solubility and dissolution of poorly soluble compounds. Table 4 demonstrates the compositions of three snapshot media and FeSSIF-V2.

It has been shown recently that the updated versions of small intestinal media, FaSSIF-V2 and FeSSIF-V2, demonstrate better in vivo predictiveness than their predecessors. The updated media predicted correctly that the oral absorption of glibenclamide tablets in the fasted and fed state is not significantly different (10), whereas with the previous versions of the media, results (Figure 2) had indicated that there would be a food effect (11).

\section{PRACTICAL CONSIDERATIONS}

For the successful prediction of in vivo drug behavior, not only does the dissolution media composition have to be considered, but also the media volume and hydrodynamics. These should be selected appropriately according to the physiological values.

With respect to media volume, the in vitro setups should preferably be tailored to the physiology, but the

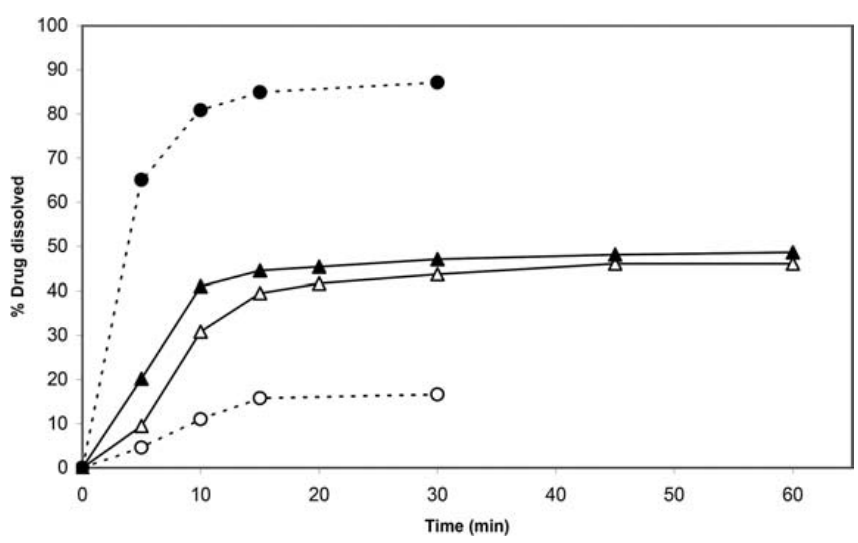

Figure 2. Dissolution profile comparison of glibenclamide tablets

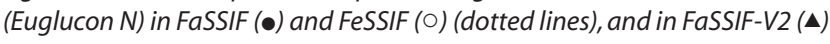
and FeSSIF-V2 $(\triangle)$ (continuous lines). (Reproduced from ref 10.)

practical constraints of the test apparatus also need to be considered. Some reports on the volume of GI fluids are available $(1,12,13)$. In summary, the fluid volume in the stomach would initially be around $300 \mathrm{~mL}$ in the fasted state and $500 \mathrm{~mL}$ or more in the fed state, while in the small intestine, volumes of $200 \mathrm{~mL}$ in the fasted and $1 \mathrm{~L}$ in the fed state appear to be reasonable.

In terms of hydrodynamics, the apparatus listed in the pharmacopeial monographs can only partly mimic the motility in the GI tract and therefore cannot be considered truly biorelevant. However, they have been used successfully to generate in vitro-in vivo correlations (IVIVC) in combination with biorelevant media, and

Table 4. Composition of the Media to Simulate the Fed-State Upper Small Intestine, Including Fed-State Simulated Intestinal Fluid, Updated Version (FeSSIF-V2)

\begin{tabular}{|c|c|c|c|c|}
\hline Composition (mM) & Early FeSSIF & Middle FeSSIF & Late FeSSIF & FeSSIF-V2 \\
\hline Sodium taurocholate & 10 & 7.5 & 4.5 & 10 \\
\hline Lecithin & 3 & 2 & 0.5 & 2 \\
\hline Glyceryl monooleate & 6.5 & 5 & 1 & 5 \\
\hline Sodium oleate & 40 & 30 & 0.8 & 0.8 \\
\hline Maleic acid & 28.6 & 44 & 58.09 & 55.02 \\
\hline Sodium hydroxide & 52.5 & 65.3 & 72 & 81.65 \\
\hline Sodium chloride & 145.2 & 122.8 & 51 & 125.5 \\
\hline \multicolumn{5}{|l|}{ Properties } \\
\hline $\mathrm{pH}$ & 6.5 & 5.8 & 5.4 & 5.8 \\
\hline Osmolality (mOsm/kg) & $400 \pm 10$ & $390 \pm 10$ & $240 \pm 10$ & $390 \pm 10$ \\
\hline Buffer capacity (mmol/L/pH) & 25 & 25 & 15 & 25 \\
\hline Surface tension (mN/m) & $30.1 \pm 0.2$ & $32.7 \pm 0.5$ & $46.0 \pm 0.2$ & $40.5 \pm 0.2$ \\
\hline
\end{tabular}


therefore have some application for this purpose.

For IVIVC purposes, appropriate justification of apparatus selection should be made on a case-by-case basis (e.g.,

(A)

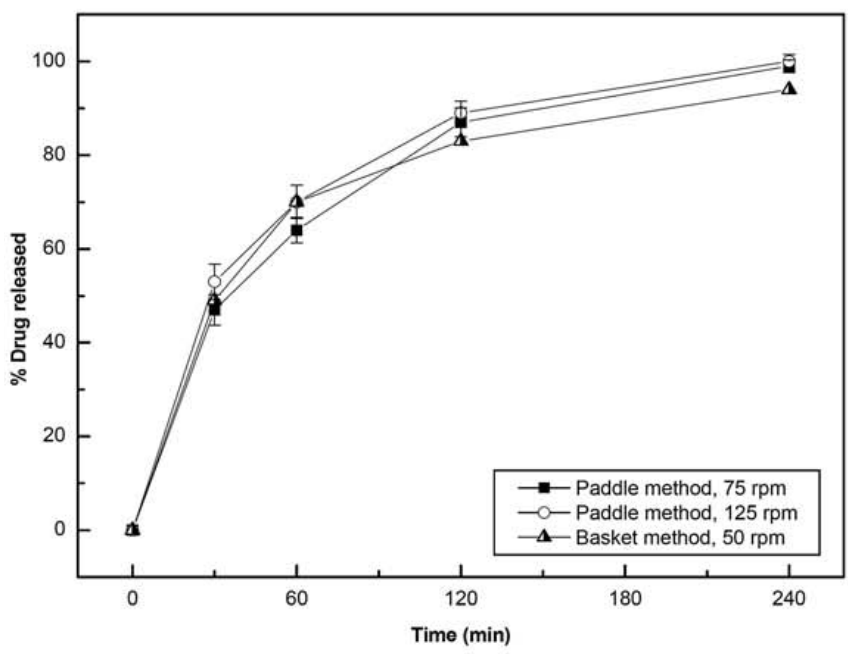

(B)

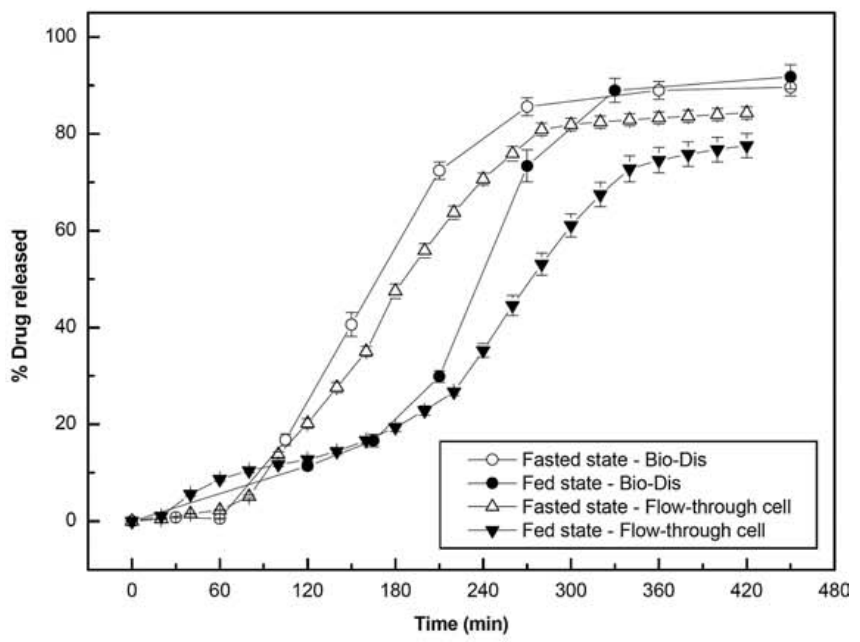

(C)

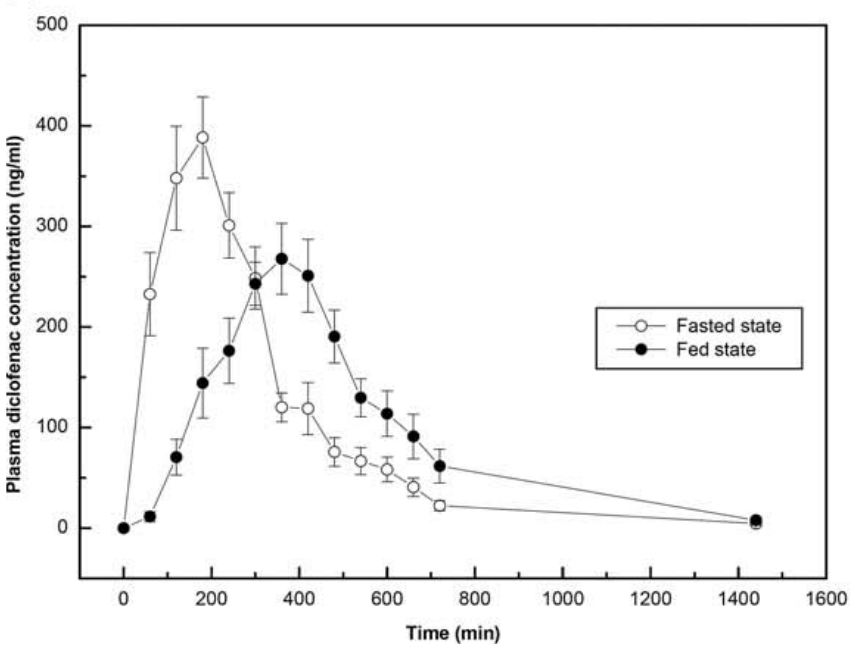

the use of flow-through apparatus to simulate sink conditions for the dissolution tests of poorly soluble, highly permeable drugs, which would be difficult to achieve with conventional paddle or basket apparatus).

For modified-release (MR) dosage forms, the use of a series of biorelevant media simulating the sequential changes in the stomach through the small intestine (or even colon) appears to be reasonable since the dosage forms will experience drastically different conditions as they move along the Gl tract. This concept has been recently applied to the investigation of prandial state effects on absorption of diclofenac sodium (Figure 3) (14). In that study, a Level A IVIVC between the in vitro dissolution and the in vivo fraction absorbed was demonstrated using two series of biorelevant media representing the fasted and fed human gut, coupled with the Bio-Dis and flow-through apparatus. If more specific questions, like How would the dosage form behave if it has a prolonged residence in the fed stomach? (a pertinent question for behavior of MR monolithic dosage forms) need to be addressed, the use of three gastric snapshot media as one series may be a useful approach.

The use of enzymes with biorelevant media, like the lipolysis-model approach, is appropriate in certain cases, for example, to assess lipid-based formulations $(15,16)$. Recently, the importance of intragastric lypolysis to the release of felodipine from a matrix tablet has also been shown (17).

\section{CONCLUDING REMARKS}

Four core biorelevant dissolution media representing the human stomach and upper small intestine have been proposed. Some have been introduced recently into the literature; others are updates of existing recipes. The media compositions closely resemble recent physiological data and reflect the digestion processes better than previous biorelevant media and much better than compendial media. These biorelevant media can be applied to assess drugs and their dosage forms during the course of drug product development.

\section{REFERENCES}

1. Dressman, J. B.; Amidon, G. L.; Reppas, C.; Shah, V.P. Dissolution testing as a prognostic tool for oral drug absorption: immediate release dosage forms. Pharm. Res. 1998, 15 (1), 11-22.

2. Kalantzi, L.; Goumas, K.; Kalioras, V.; Abrahamsson, B.; Dressman, J. B., Reppas, C. Characterization of the

Figure 3. Comparison of cumulative diclofenac sodium dissolution-versustime profiles using $(A)$ the QC methods (basket and paddle) and (B) the biorelevant methods in the fasted-and fed-state using the Bio-Dis and flow-through apparatus. (C) Plasma diclofenac sodium concentration-time profiles obtained following a single oral administration of modified-release diclofenac sodium pellets to 16 healthy volunteers in the fasted and fed states. (Reproduced with permission from ref 14. Copyright 2009 Elsevier.) 
human upper gastrointestinal contents under conditions simulating bioavailability/bioequivalence studies. Pharm. Res. 2006, 23 (1), 165-176.

3. Porter, C. J.; Trevaskis, N. L.; Charman, W. N. Lipids and lipid-based formulations: optimizing the oral delivery of lipophilic drugs. Nat. Rev. Drug Discov. 2007, 6 (3), 231-248.

4. Jantratid, E.; Janssen, N.; Reppas, C.; Dressman, J. B. Dissolution media simulating conditions in the proximal human gastrointestinal tract: an update. Pharm. Res. 2008, 25 (7), 1663-1676.

5. Vertzoni, M.; Dressman, J.; Butler, J.; Hempenstall, J.; Reppas, C. Simulation of fasting gastric conditions and its importance for the in vivo dissolution of lipophilic compounds. Eur. J. Pharm. Biopharm. 2005, 60 (3), 413-417.

6. Dressman, J. B.; Berardi, R. R.; Dermentzoglou, L. C.; Russell, T. L.; Schmaltz, S. P.; Barnett, J. L.; Jarvenpaa, K. M. Upper gastrointestinal (Gl) pH in young, healthy men and women. Pharm. Res. 1990, 7 (7), 756-761.

7. United States Pharmacopeia and National Formulary USP 31-NF 26; The United States Pharmacopeial Convention, Inc.: Rockville, MD, 2008.

8. Dressman, J. B.; Vertzoni, M.; Goumas, K.; Reppas, C. Estimating drug solubility in the gastrointestinal tract. Adv. Drug Deliv. Rev. 2007, 59 (7), 591-602.

9. Jantratid, E.; Janssen, N.; Chokshi, H.; Tang, K.; Dressman, J. B. Designing biorelevant dissolution tests for lipid formulations: case example-lipid suspension of RZ-50. Eur. J. Pharm. Biopharm. 2008, 69 (2), 776-785.

10. Janssen, N.; Jantratid, E.; Dressman, J. B. Influence of biorelevant media compositions on the dissolution behavior of glibenclamide tablets. Proceedings of the 6th World Meeting on Pharmaceutics, Biopharmaceutics, and Pharmaceutical Technology, Barcelona, Spain, April 7-10, 2008.

11. Löbenberg, R.; Krämer, J.; Shah, V. P.; Amidon, G. L.; Dressman, J. B. Dissolution testing as a prognostic tool for oral drug absorption: dissolution behavior of glibenclamide. Pharm. Res. 2000, 17 (4), 439-444.

12. Welling, P. G. Effects of food on drug absorption. Annu. Rev. Nutr. 1996, 16, 383-415.

13. Custodio, J. M.; Wu, C. Y.; Benet, L. Z. Predicting drug disposition, absorption/elimination/transporter interplay and the role of food on drug absorption. Adv. Drug Deliv. Rev. 2008, 60 (6), 717-733.

14. Jantratid, E.; De Maio, V.; Ronda, E.; Mattavelli, V.; Vertzoni, M.; Dressman, J. B. Application of biorelevant dissolution tests to the prediction of in vivo performance of diclofenac sodium from an oral modified-release pellet dosage form. Eur. J. Pharm. Sci. 2009, 37 (3-4), 434-441.

15. Zangenberg, N. H.; Müllertz, A.; Kristensen, H. G.; Hovgaard, L. A dynamic in vitro lipolysis model. I. Controlling the rate of lipolysis by continuous addition of calcium. Eur. J. Pharm. Sci. 2001, 14 (2), 115-122.

16. Zangenberg, N. H.; Müllertz, A.; Kristensen, H. G.; Hovgaard, L. A dynamic in vitro lipolysis model. II: Evaluation of the model. Eur. J. Pharm. Sci. 2001, 14 (3), 237-244.

17. Diakidou, A.; Vertzoni, M.; Abrahamsson, B.; Dressman, J.; Reppas, C. Simulation of gastric lipolysis and prediction of felodipine release from a matrix tablet in the fed stomach. Eur. J. Pharm. Sci. 2009, 37 (2), 133-140.

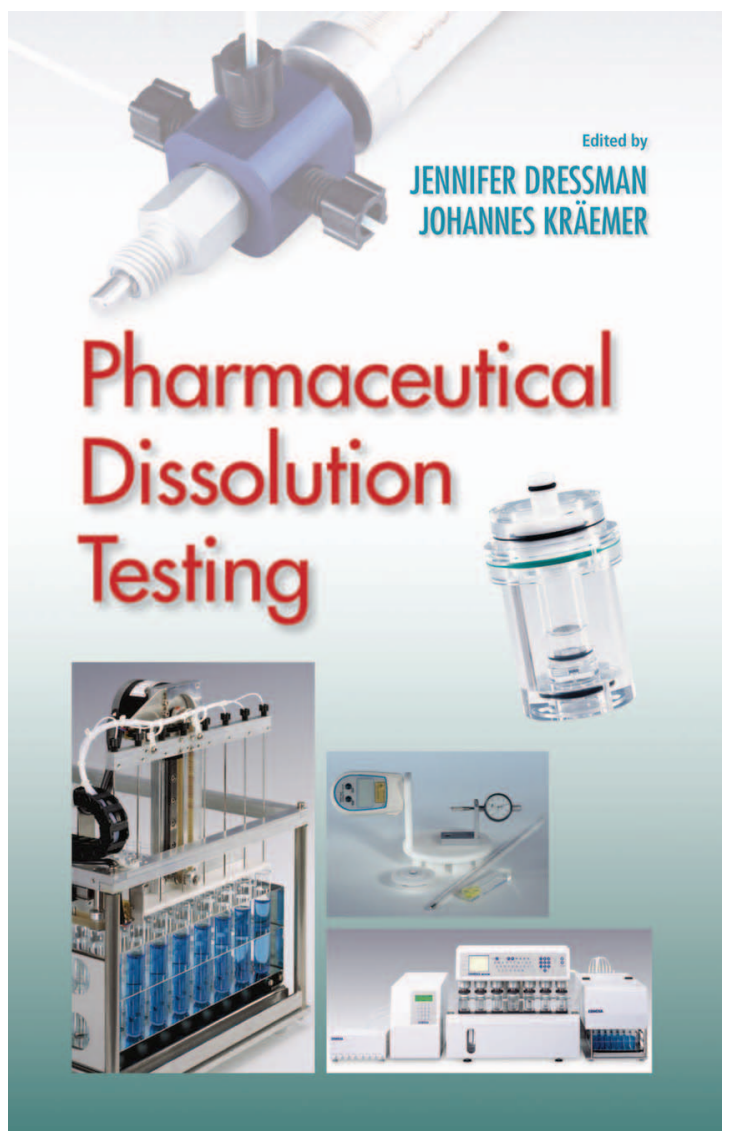

This book may be ordered online at the DT website at www.dissolutiontech.com. Price, including shipping, is $\$ 210.00$ for US and $\$ 230.00$ for outside USA. Credit card payment is available. 